\title{
Canine Hepatocellular Carcinoma
}

National Cancer Institute

\section{Source}

National Cancer Institute. Canine Hepatocellular Carcinoma. NCI Thesaurus. Code C129298.

A malignant tumor arising from hepatocytes that occurs in dogs. 